\title{
DESENVOLVIMENTO HUMANO E DESIGUALDADE REGIONAL: UMA DISCUSSÃO PARA O ESTADO DE RONDÔNIA.
}

\author{
Cleiton Franco', Karine Medeiros Anunciato
}

\section{RESUMO}

O presente estudo tem o propósito de analisar o painel dos indicadores sociais para o estado de Rondônia. A orientação teórica utilizada na discussão envolve a multidimensionalidade da pobreza e aspectos da desigualdade social. Foi delimitado como corte analítico os anos de 2001 a 2011 dos dados da Pesquisa Nacional por Amostra de Domicílios (PNAD), do Censo Demográfico de 2010 do IBGE e do Atlas do Desenvolvimento Humano no Brasil de 2013. Em 2010, Rondônia apareceu com $15^{\circ}$ colocado no ranking dos estados brasileiros com IDH de 0,69 . A proporção de pobres no estado tem apresentado redução, resultando em menor valor indicativo no ano de 2011 (19,60\%) consideravelmente menor que 2003 (44,85\%). No ano de 2010 apenas 49, dos 52 municípios, apresentaram Índice de Gini maior ou igual a 0,50. Palavras-chave: indicadores sociais; políticas sociais; IDH; pobreza.

\section{INTRODUÇÃO}

O Brasil ocupa hoje, em termos de desigualdade social, a 116 . Posição na lista de países com menor índice de desigualdade social, atrás, por exemplo, da Etiópia, atualmente na $11^{\mathrm{a}}$. posição. A lista é liderada por países como Dinamarca, Japão e Suécia.

A Pesquisa Nacional por Amostra de Domicílios (Pnad 2011) apresenta resultados que confirmam que a primeira década do século 21 no Brasil foi "inclusiva" do ponto de vista social, com robusta diminuição da desigualdade e redução da pobreza, na avaliação do Instituto de Pesquisa Econômica Aplicada (Ipea). Este período apresenta os melhores resultados desde o início quando o país passou a informar estatísticas sobre distribuição de renda.

O índice de Gini (indicador que mede a desigualdade) foi 0,527 em 2011 - o menor desde $1960(0,535)$ - quanto mais próximo de zero menor é a desigualdade. Esta redução se deve ao crescimento da renda per capita nos diferentes estratos sociais. Entre 2001 e 2011, o crescimento real da renda dos $10 \%$ mais pobres foi $91,2 \%$. Enquanto que para os $10 \%$ mais ricos, o crescimento foi $16,6 \%$. Na opinião do Ipea, a melhoria da renda na base da pirâmide relativiza o tímido desempenho das contas nacionais (medido pelo Produto Interno Bruto - PIB).

Nas contas do Ipea, as transferências do Programa Bolsa Família são responsáveis por $13 \%$ da redução da desigualdade. O efeito é gerado pela renda que cresce mais entre os menos escolarizados, os pretos e pardos, as crianças de até 4 anos, a população do Nordeste e os residentes em áreas rurais, o que numa análise histórica, são os setores mais pobres da sociedade brasileira.

\footnotetext{
${ }^{1}$ Universidade do Estado de Mato Grosso - UNEMAT

${ }^{2}$ Universidade do Estado de Mato Grosso - UNEMAT
} 
Apesar dos bons resultados, a análise do Ipea sobre a Pnad (2011) mostra que a renda está crescendo nos setores econômicos que contratam mão de obra de forma precária e agregam pouco valor à economia, como a agricultura $(86 \%)$ e as atividades domésticas $(62,4 \%)$. Outro dado preocupante é que 35\% da diminuição da desigualdade se devem aos repasses feitos pelo governo (Bolsa Família, aposentadorias, pensões e benefícios de prestação continuada). Essas transferências estão sujeitas à política fiscal (que pode ser restritiva para que as contas públicas tenham superávit).

A literatura envolvendo a discussão a cerca de indicadores sociais, desigualdade de renda e pobreza, buscando atender a diferentes objetivos pode ser encontrada nos trabalhos de ...

O objetivo deste trabalho foi analisar o painel dos indicadores sociais envolvendo os municípios do estado de Rondônia, considerando aspectos de IDH, desigualdade de renda e inidice de Gini. Buscou-se caracterizar o contexto social, econômico e político.

\section{INDICADORES SOCIAIS}

Indicadores são medidas de comportamento e do desenvolvimento de sistemas complexos que fornecem uma confiável síntese dos resultados identificados. As relações entre o padrão de respostas e do conjunto de indicadores de sistemas permite a previsão de condições futuras. Devem indicar por meio de medidas as modificações que podem ocorrer em um determinado ambiente devido às mudanças determinadas pela ação antrópica. (MARZALL, 2000)

Os indicadores, dessa forma, seriam medidas dos processos de produção, que podem servir como resumo de resultados de ambientes complexos, de maneira a contribuir para o desenvolvimento de políticas que possam colaborar na redução da desigualdade social e pobreza, uma vez que o principal usuário é o próprio governo, como também, empresas que buscam estratégias de sustentabilidade e consumidores que se preocupam com questões relacionadas as atividades econômicas.

Para atingir uma dada utilidade, um indicador necessita ser confiável, representativo e comparável permitindo com isso, explicações de causas de mudanças ao longo do tempo. Além disso, deve ser suficientemente simples à medida que descreve problemas complexos, usando definições comuns e normas que permitam comparações (COLE 2002).

Segundo Borba (2009) o indicador é um estimador relacionado a um atributo, propriedade ou característica determinada, que irá fornecer informações sobre o 
comportamento do fenômeno ou sistema objeto do estudo. Indicadores podem ser derivados de outras variáveis, sendo importante o entendimento da relação com dados primários.

Quanto às funções dos indicadores estes podem ser divididos em indicadores sistêmicos e de performance. Os sistêmicos estão baseados em referenciais técnicos e possuem a capacidade de comunicar as informações mais importantes aos tomadores de decisão. Os de performance fornecem informações sobre o grau de sucesso na realização das metas, sendo bastante utilizados no campo da avaliação política e no processo decisório (HARDI E BARG, 1997).

$\mathrm{Na}$ pesquisa acadêmica, o indicador de caráter social pode servir como elo entre modelos explicativos da Teoria Social e a evidencia empírica dos fenômenos sociais observados. Em uma perspectiva programática, o Indicador Social torna-se instrumento operacional para monitoramento da realidade social, para fins de formulação e reformulação de politicas publicas. Taxas de analfabetismo, rendimento médio do trabalho, taxas de mortalidade infantil, taxas de desemprego, Índice de Gini, proporção de crianças matriculadas em escolas são, neste sentido, indicadores sociais, ao traduzir em cifras tangíveis e operacionais das varias dimensões relevantes, especificas e dinâmicas da realidade social (JANNUZZI, 2001).

Classificam-se os indicadores entre objetivos e subjetivos, ou quantitativos e qualitativos. Os indicadores objetivos (de caráter quantitativo) são construídos à partir das estatísticas disponíveis em fontes públicas e se referem a ocorrências concretas ou estudos empíricos da realidade social. Os exemplos podem ser encontrados na taxa de desemprego, evasão escolar ou o risco em acidentes de trabalho. Indicadores subjetivos (de caráter qualitativo) são medidas construídas a partir da avaliação de indivíduos ou especialistas em relação a aspectos da realidade, identificadas geralmente em pesquisas de opinião pública ou em grupos de discussão (JANNUZZI, 2001).

As diferenças conceituais entre indicadores quantitativos e qualitativos podem gerar conclusões diferenciadas quanto à mesma dimensão social. Em investigações dos impactos sociais de programas do governo, por exemplo, é possível que indicadores objetivos reflitam melhorias relacionadas às condições materiais de vida da população que, de outra forma, não apresentam indicadores de satisfação considerados favoráveis, quando baseados na opinião dos entrevistados. 
Indicadores de impacto referente à exclusão social e sistemas de avaliação voltados às demandas dos tomadores de decisão, e anseios da sociedade são necessários para gestão das cidades e que contribuem no processo de monitoramento das desigualdades sociais.

\section{METODOLOGIA}

\subsection{O Índice de Desenvolvimento Humano (IDH)}

O Índice de Desenvolvimento Humano (IDH) é uma medida concebida pela Organização das Nações Unidas (ONU) com a finalidade de avaliar a qualidade de vida e o desenvolvimento econômico de uma população. A referência principal trata-se do Atlas do Desenvolvimento Humano. No Brasil, tornou-se uma plataforma de consulta ao Índice de Desenvolvimento Humano Municipal (IDHM) de 5.565 municípios brasileiros de mais de 180 indicadores de população, educação, habitação, saúde, trabalho, renda e vulnerabilidade, com dados extraídos dos Censos Demográficos de 1991, 2000 e 2010.

O índice varia de 0 (nenhum desenvolvimento humano) a 1 (desenvolvimento humano total). Segundo PNUD (2013) nações com IDH até 0,499 têm desenvolvimento humano considerado baixo; os índices entre 0,500 e 0,799 são considerados de médio desenvolvimento humano; IDH maior que 0,800 têm desenvolvimento humano considerado alto.

A fim de analisar as condições de núcleos sociais menores, como os municípios utiliza-se o IDH municipal (IDHM), onde são considerados fatores como educação, longevidade e renda. As três dimensões analisadas são: IDHM-E, para educação; IDHM-L, para longevidade e IDHM-R, para renda. Para tanto, são determinados os valores de referência para mínimo e máximo de cada categoria, que serão equivalentes a 0 e 1 , respectivamente, no cálculo do índice. Os subíndices de cada município serão valores proporcionais dentro dessa escala: quanto melhor o desempenho municipal naquela dimensão, mais próximo o seu índice estará de 1. O IDHM de cada município é dado por:

$$
I D H M=\frac{(\mathrm{IDHM}-\mathrm{E}+\mathrm{IDHM}-\mathrm{L}+\mathrm{IDHM}-\mathrm{R})}{3}
$$




\subsection{O Índice de GINI}

Trata-se do índice de distribuição de renda mais conhecido e de maior aplicação (NEDER, 2013),. O Gini é uma medida de desigualdade utilizada comumente para calcular a desigualdade de distribuição de renda, podendo também ser usada para qualquer distribuição, como concentração de riqueza, de terra, entre outras (IPECE, 2010).

Consiste em um número entre 0 e 1 , onde 0 corresponde à completa igualdade de renda, onde todos têm a mesma renda e 1 corresponde à completa desigualdade, onde uma pessoa tem toda a renda, e as demais nada têm. Dessa forma quanto mais o índice tende a zero, melhor será a distribuição de renda, quanto mais próximo de um, pior a distribuição e a desigualdade.

Seguindo a metodologia de Costa (2010) e IPECE (2010), considerando variável aleatória discreta $X_{i}=(i=1, \ldots, n)$, cujos valores estão em ordem crescente, $X_{1} \leq X_{2} \leq$ $X_{3} \leq \cdots \leq X_{n-1} \leq X_{n}$. Supondo que os valores de $X$ sejam igualmente prováveis.

A proporção acumulada do número de elementos até o i-ésimo é:

$P_{i}=\frac{i}{n}(i, \ldots, n)$

A correspondente acumulada de $\mathrm{X}$, até o i-ésimo elemento é:

$\phi_{i}=\frac{\sum_{j=1}^{i} X_{j}}{\sum_{j=1}^{n} X_{j}}=\frac{1}{n \mu} \sum_{j=1}^{i} X_{j}$, uma vez que $\sum_{j=1}^{n} X_{j}=n \mu$

Assim, se $X$ representa a renda individual e se $X_{i}<X_{i+1}, \phi_{i}$ representa a fração da renda total, apropriada pelos indivíduos com renda inferior ou igual a $X_{i}$. Com as equações (5) e (6) definem as coordenadas $\left(\rho_{i}, \phi_{i}\right), \operatorname{com} i=1 \ldots n$, pontos de $n$ da curva de Lorenz.

Calculando o índice de Gini para os valores discretos, segundo IPECE (2010), seja $\beta$ a área entre a curva de Lorenz e o eixo das abscissas. Assim:

$\alpha+\beta=0,5$ ou $\alpha=0,5-\beta$

Como por definição o coeficiente de Gini é uma relação entre a área da desigualdade, indicada por $\alpha$ e a área do triângulo. Tem-se:

$G=\frac{\alpha}{0,5}=2 \alpha$

Substituindo (8) em (7), tem-se:

$G=1-2 \beta$

Observa-se que a área $\beta$ pode ser obtida somando a área de $n$ trapézios. No caso do i-ésimo trapézio, tem-se a área $\delta_{i}$ sendo dada por: 
$\delta_{i}=\frac{1}{2 n}\left(\phi_{i-1}+\phi_{i}\right)$

Descartando o primeiro triângulo, ou seja, fazendo $\phi_{0}=0$, tem-se o índice de Gini:

$G=1-\frac{1}{n} \sum_{i=1}^{n}\left(\phi_{i-1}+\phi_{i}\right)$

\section{RESULTADOS E DISCUSSÃO}

Dados da Pesquisa Nacional por Amostra de Domicílios (PNAD) demonstram que o estado de Rondônia totaliza 1.601.500 milhões em 2013, distribuídos em 52 municípios, desses, apenas 17 possuem população superior a 20 mil habitantes. Composto por grandes propriedades possui áreas com vazios populacionais, resultando em baixa densidade demográfica. A população é bem distribuída em se tratando do gênero. Para o período de 2011, homens apresentam ligeira maioria da população $51 \%$.

A população de Rondônia tem crescido nesta última década mais para idades avançadas. Na última década a população situava-se na faixa etária de 20 a 29 anos, representado 19\% da população total. Para o ano de 2011 o que se observa é uma redução da população mais jovem de 0 a 19 anos (-17\% e -1\%, respectivamente), aumento moderado da população economicamente ativa dos 20 aos 40 (16\% e 21\%, respectivamente) e intensivo aumento da população mais idosa, dos 50 aos 60 (64\% e 56\%, respectivamente). O grande desafio para a população de jovens nas várias regiões do estado são os acessos à educação e geração de postos de trabalhos.

\subsection{IDHM}

Observa-se na verdade certa evolução no que diz respeito ao IDH de Rondônia (RO), isto porque em 1991 apesar de ocupar a $1^{\circ}$ posição no ranking nacional apresentava índice considerado muito baixo, de apenas 0,407. Esse índice foi melhorado em 2000, com valor de 0,537, considerado de médio desenvolvimento humano, apesar de apresenta melhoria na posição, situando-se na $17^{a}$ posição nacional. No mais recente IDH dos estados, o de 2010, Rondônia aparece na $15^{\circ}$ colocado no ranking dos estados brasileiros com IDH de 0,69, atrás dos estados vizinhos da região norte como Amapá $(0,708)$, Roraima $(0,707)$ e Tocantins $(0,699)$, já sendo considerado estado com alto desenvolvimento humano.

Com relação ao IDHM dos municípios, para o ano de 2010, os oito municípios que apresentam IDHM maiores que o índice do Estado de Rondônia são: Porto Velho (0,736), 
neste caso a capital, Vilhena (0,731), Cacoal (0,718), Ji-Paraná (0,714) Pimenta Bueno $(0,710)$, Ariquemes $(0,702)$ Rolim de Moura $(0,7)$ e Cerejeiras $(0,692)$.

\section{Quadro 01 - Os dez melhores e os 10 piores municípios classificados de acordo com o} IDHM de Rondônia em 2010

\begin{tabular}{|c|c|c|c|c|c|}
\hline Ranking & Municipios & IDHM & IDHM E & IDHM_L & IDHM_R \\
\hline 10. & PORTO VELHO & 0,736 & 0,638 & 0,819 & 0,764 \\
\hline 20. & VILHENA & 0,731 & 0,659 & 0,808 & 0,734 \\
\hline 3o. & CACOAL & 0,718 & 0,620 & 0,821 & 0,727 \\
\hline 4o. & JI-PARANÁ & 0,714 & 0,617 & 0,810 & 0,728 \\
\hline 50. & PIMENTA BUENO & 0,710 & 0,613 & 0,803 & 0,726 \\
\hline 60. & ARIQUEMES & 0,702 & 0,600 & 0,806 & 0,716 \\
\hline 7o. & ROLIM DE MOURA & 0,700 & 0,598 & 0,808 & 0,709 \\
\hline 80. & CEREJEIRAS & 0,692 & 0,602 & 0,799 & 0,688 \\
\hline 9o. & JARU & 0,689 & 0,577 & 0,825 & 0,687 \\
\hline 100. & COLORADO DO OESTE & 0,685 & 0,584 & 0,814 & 0,676 \\
\hline ... & $\ldots$ & $\ldots$ & $\ldots$ & $\ldots$ & \\
\hline 430. & MONTE NEGRO & 0,607 & 0,454 & 0,762 & 0,645 \\
\hline 440. & SERINGUEIRAS & 0,598 & 0,427 & 0,779 & 0,644 \\
\hline 45o. & MACHADINHO D'OESTE & 0,596 & 0,446 & 0,755 & 0,629 \\
\hline 46o. & GOVERNADOR JORGE TEIXEIRA & 0,596 & 0,444 & 0,762 & 0,627 \\
\hline 47o. & CAMPO NOVO DE RONDÔNIA & 0,593 & 0,404 & 0,772 & 0,667 \\
\hline 480. & ALTO ALEGRE DOS PARECIS & 0,592 & 0,443 & 0,777 & 0,603 \\
\hline 49o. & THEOBROMA & 0,589 & 0,434 & 0,757 & 0,622 \\
\hline 500. & NOVA MAMORÉ & 0,587 & 0,424 & 0,769 & 0,619 \\
\hline 510. & NOVA UNIÃO & 0,587 & 0,442 & 0,753 & 0,608 \\
\hline 520. & VALE DO ANARI & 0,584 & 0,421 & 0,756 & 0,626 \\
\hline
\end{tabular}

Fonte: Atlas do Desenvolvimento Humano no Brasil, 2013.

A respeito das dimensões que compõem o IDH do estado em 2010 são: IDHM-R de 0,712, IDHM-E de 0,577 e o IDHM-L com 0,8. O município que mais se destaca com relação à renda é Porto Velho, com índice de 0,764 . Contudo com relação à longevidade (saúde) o município de Jaru destaca-se com índice de 0,825. Já em relação a educação o município destaque é Vilhena, com índice de 0,659 (QUADRO 1).

Considerando ainda os demais IDHM, que possuem faixas de desenvolvimento humano consideradas baixas, ou seja, situadas entre 0,500 e 0,599, podemos destacar os municípios de Vale do Anari, Nova União, Nova Mamoré, Theobroma, Alto Alegre dos Parecis, Campo Novo de Rondônia, Governador Jorge Teixeira e Machadinho d'oeste com os piores resultados. Estes municípios são considerados ainda na dimensão da educação, como os piores resultados, resultando em índices abaixo de 0,499 (QUADRO 1). 
Quadro 02 - Matriz de correlação entre os valores do IDHM e de seus componentes para os municípios do Estado de Rondônia

\begin{tabular}{|c|c|c|c|c|c|c|c|c|c|c|c|c|}
\hline & IDHM1991 & IDHM2000 & IDHM2010 I & IDHM-E1991 & IDHM-E2000 & IDHM-E2010 & IDHM-L1991 & 1 IDHM-L2000 & IDHM-L2010 & IDHM-R1991 & IDHM-R2000 & IDHM-R2010 \\
\hline IDHM1991 & 1,000 & & & & & & & & & & & \\
\hline IDHM2000 & 0,8660 & 1,000 & & & & & & & & & & \\
\hline IDHM2010 & 0,7103 & 0,8993 & 1,000 & & & & & & & & & \\
\hline IDHM-E1991 & 0,9843 & 0,8567 & 0,6997 & 1,000 & & & & & & & & \\
\hline IDHM-E2000 & 0,8254 & 0,9788 & 0,8734 & 0,8323 & 1,000 & & & & & & & \\
\hline IDHM-E2010 & 0,6605 & 0,8593 & 0,9669 & 0,6623 & 0,8519 & 1,000 & & & & & & \\
\hline IDHM-L1991 & 0,4824 & 0,5612 & 0,5409 & 0,4222 & 0,5213 & 0,4888 & 1,000 & & & & & \\
\hline IDHM-L2000 & 0,5577 & 0,7446 & 0,7438 & 0,5128 & 0,6749 & 0,6534 & 0,6293 & 1,000 & & & & \\
\hline IDHM-L2010 & 0,3580 & 0,5950 & 0,7199 & 0,3452 & 0,5777 & 0,5972 & 0,3631 & 0,7151 & 1,000 & & & \\
\hline IDHM-R1991 & 0,8843 & 0,7615 & 0,5967 & 0,8454 & 0,7143 & 0,5192 & 0,4754 & 0,5778 & 0,3706 & 1,000 & & \\
\hline IDHM-R2000 & 0,6918 & 0,6275 & 0,5529 & 0,6642 & 0,4797 & 0,4672 & 0,3643 & 0,5043 & 0,2352 & 0,6234 & 1,000 & \\
\hline IDHM-R2010 & 0,7658 & 0,8389 & 0,8684 & 0,7360 & 0,7763 & 0,7494 & 0,5551 & 0,6929 & 0,5657 & 0,6819 & 0,7355 & 1,000 \\
\hline
\end{tabular}

Observe que os IDHM 2000 e o IDHM 2010 têm elevada correlação $(0,8993)$ indicando que os municípios conservaram as mesmas colocações durante este período. A mesma situação não ocorre com os IDHM 1991 e IDHM 2010, cuja correlação foi de 0,8660. Esse fator é função do fato de que RO estaria em $19^{\circ}$ no ranking nacional em 1991 , porém por apresentar resultados ruins em seu IDH, e ao subir para a $15^{\circ} \mathrm{em} 2010$ melhorar seu desempenho de maneira geral (QUADRO 2).

Este resultado se confirma pelo resultado do coeficiente de correlação de Sperman (correlação de ordem) é de 0,8434 entre o IDHM de 2000 e 2010, e de apenas 0,7373 entre o IDHM de 1991 e 2000. Observa-se também a baixa correlação entre os IDHM de 1991 como os IDMH de 2000 e de IDHM de 2010 de educação, renda e longevidade (QUADRO $3)$.

\section{Quadro 3 - Coeficientes de correlação de Spearman entre os diversos componentes do}

IDH e para os municípios do Estado de Rondônia

\begin{tabular}{|c|c|c|c|c|c|c|c|c|c|}
\hline IDHM-E1991 & 1,0000 & & & & & & & & \\
\hline IDHM-E2000 & 0,7373 & 1,0000 & & & & & & & \\
\hline IDHM-E2010 & 0,5894 & 0,8434 & 1,0000 & & & & & & \\
\hline IDHM-L1991 & 0,3987 & 0,5061 & 0,4721 & 1,0000 & & & & & \\
\hline IDHM-L2000 & 0,4855 & 0,6584 & 0,6645 & 0,6214 & 1,0000 & & & & \\
\hline IDHM-L2010 & 0,3194 & 0,5867 & 0,5979 & 0,3961 & 0,7726 & 1,0000 & & & \\
\hline IDHM-R1991 & 0,7715 & 0,6130 & 0,4235 & 0,4906 & 0,5542 & 0,3736 & 1,0000 & & \\
\hline IDHM-R2000 & 0,6482 & 0,3872 & 0,4276 & 0,3970 & 0,5397 & 0,2473 & 0,6144 & 1,0000 & \\
\hline IDHM-R2010 & 0,6920 & 0,6579 & 0,6569 & 0,5711 & 0,7202 & 0,5912 & 0,5661 & 0,7103 & 1,0000 \\
\hline
\end{tabular}




\subsection{Indicadores de pobreza}

Identificar a proporção de pobres em determinada região é importante para subsidiar processos de planejamento, gestão e avaliação de políticas de distribuição de renda (NEDER, 2013). Os dados do Censo de 2010 apontam que 121.290 pessoas estariam vivendo em situação de extrema pobreza no estado de Rondônia. $O$ quantitativo de pessoas nesta condição é 56.064 na zona urbana, e o restante, 65.226 reside na zona rural. A faixa de idade mais afetada pela pobreza extrema no estado é a de 20 a 39 anos com 28,84\% das pessoas pobres. Outro ponto importante é que $49 \%$ da população pobre no estado tem menos de 19 anos, o que compromete o desenvolvimento individual e deixa sequelas na educação e na saúde.

A proporção de pessoas que vivem na extrema pobreza tem sido reduzida ao longo dos anos. Dentre os municípios que ainda apresentam índices de pobreza extrema nos anos de 1991, 2000 e 2010 destacam-se São Miguel do Guaporé e Alto Alegre dos Parecis. Já os municípios de Vilhena, Porto Velho, Ji-Paraná e Ouro Preto do Oeste aparecem nos referidos anos dentre os dez municípios com as menores proporções percentuais de pobreza extrema (QUADRO 4).

Quadro 04 - Os dez menores e maiores municípios classificados por percentual de pobreza extrema*, em Rondônia para os anos de 1991, 2000 e 2010.

\begin{tabular}{|c|c|c|c|c|}
\hline Classif. & Municipio & 1991 Municipio & 2000 Municipio & 2010 \\
\hline 10. & VILHENA & 6,65 VILHENA & 5,16 VILHENA & 2,10 \\
\hline 20. & PORTO VELHO & 8,40 JI-PARANÁ & 6,50 PIMENTA BUENO & 2,29 \\
\hline 30. & GUAJARÁ-MIRIM & 14,84 SÃO FRANCISCO DO GUAPORÉ & 6,94 PORTO VELHO & 2,64 \\
\hline 40. & PIMENTA BUENO & 15,15 CHUPINGUAIA & 7,09 JI-PARANÁ & 2,67 \\
\hline 50. & BURITIS & $15,61 \mathrm{CACOAL}$ & 7,76 OURO PRETO DO OESTE & 3,71 \\
\hline 60. & JI-PARANÁ & 16,70 PORTO VELHO & 7,81 ROLIM DE MOURA & 3,96 \\
\hline 70. & ARIQUEMES & 18,90 ARIQUEMES & 8,01 JARU & 4,32 \\
\hline 80. & OURO PRETO DO OESTE & 21,39 ROLIM DE MOURA & 9,98 ARIQUEMES & 4,36 \\
\hline 90. & CACOAL & 24,76 PIMENTA BUENO & 10,70 CEREJEIRAS & 4,72 \\
\hline 100. & CHUPINGUAIA & 25,51 OURO PRETO DO OESTE & 11,26 CHUPINGUAIA & 5,39 \\
\hline$\cdots$ & $\cdots$ & $\cdots \quad \ldots$ & & $\cdots$ \\
\hline 430. & SÃO FELIPE D'OESTE & 52,35 VALE DO PARAÍSO & 24,56 CAMPO NOVO DE RONDÔNIA & 42 \\
\hline 440. & MINISTRO ANDREAZZA & 52,60 ALVORADA D'OESTE & 24,57 VALE DO PARAÍSO & 43 \\
\hline 450. & ALTO ALEGRE DOS PARECIS & 53,82 NOVA UNIÃO & 24,65 PARECIS & 44 \\
\hline 460. & SANTA LUZIA D'OESTE & 55,74 MACHADINHO D'OESTE & 26,91 SERINGUEIRAS & 45 \\
\hline 47o. & SÃO MIGUEL DO GUAPORÉ & 55,87 URUPÁ & 27,80 NOVO HORIZONTE DO OESTE & 46 \\
\hline 480. & SERINGUEIRAS & 57,99 CASTANHEIRAS & 27,82 RIO CRESPO & 47 \\
\hline 490. & CASTANHEIRAS & 58,29 CAMPO NOVO DE RONDÔNIA & 28,40 VALE DO ANARI & 48 \\
\hline 500. & CABIXI & 60,76 THEOBROMA & 29,46 MACHADINHO D'OESTE & 49 \\
\hline 510. & VALE DO ANARI & 65,98 NOVA MAMORÉ & 30,53 THEOBROMA & 50 \\
\hline 520. & CORUMBIARA & 67,29 PARECIS & 33,56 ALTO ALEGRE DOS PARECIS & 51 \\
\hline 530. & NOVO HORIZONTE DO OESTE & 68,10 RIO CRESPO & 41,11 SÃO MIGUEL DO GUAPORÉ & 52 \\
\hline
\end{tabular}

* Proporção dos indivíduos com renda domiciliar per capita mensal igual ou inferior a $\mathrm{R} \$ 70,00$ mensais.

Fonte: Atlas do Desenvolvimento Humano no Brasil, 2013. 


\section{3 Índice de GINI}

È um cálculo cujo objetivo é medir o grau de concentração de renda em determinados grupos. Identifica a diferença entre os rendimentos dos mais pobres e dos mais ricos. Largamente usado em diversos estudos relacionados à desenvolvimento econômico, distribuição de renda, bem estar social e pobreza. Este índice possibilita a observação nos últimos anos, da distância que separa os mais ricos na base da pirâmide dos mais pobres caiu no Brasil e na região norte (QUADRO 5).

Quadro 5 - Coeficiente de Gini para a Região Norte do Brasil de 2001 a 2011.

\begin{tabular}{lrrrrrrrrrr}
\hline Estados & 2011 & 2009 & 2008 & 2007 & 2006 & 2005 & 2004 & 2003 & 2002 & 2001 \\
\hline Brasil & 0,529 & 0,544 & 0,544 & 0,553 & 0,56 & 0,567 & 0,57 & 0,581 & 0,587 & 0,594 \\
Rondonia & 0,496 & 0,506 & 0,498 & 0,505 & 0,543 & 0,568 & 0,518 & 0,505 & 0,541 & 0,548 \\
Acre & 0,545 & 0,61 & 0,56 & 0,608 & 0,592 & 0,585 & 0,594 & 0,579 & 0,623 & 0,628 \\
Amazonas & 0,541 & 0,508 & 0,514 & 0,551 & 0,511 & 0,513 & 0,537 & 0,558 & 0,563 & 0,576 \\
Roraima & 0,524 & 0,524 & 0,543 & 0,516 & 0,565 & 0,542 & 0,584 & 0,525 & 0,56 & 0,543 \\
Para & 0,539 & 0,509 & 0,497 & 0,52 & 0,506 & 0,516 & 0,537 & 0,519 & 0,56 & 0,553 \\
Amapa & 0,523 & 0,518 & 0,454 & 0,509 & 0,478 & 0,526 & 0,542 & 0,599 & 0,551 & 0,482 \\
Tocantins & 0,520 & 0,521 & 0,542 & 0,545 & 0,521 & 0,536 & 0,552 & 0,565 & 0,562 & 0,599 \\
\hline
\end{tabular}

Fonte: Calculado da PNAD

Ao analisar o Índice de Gini tem-se em mente que o valor zero representa a situação de igualdade total, ou seja, todos teriam a mesma renda. No extremo oposto seria o valor um, ou seja, só uma pessoa detém toda a riqueza. Do período de 2001 a 2011 os valores do Gini para o estado de Rondônia não variaram significativamente, o que situa o estado em posição privilegiada quanto à distribuição de renda da população.

Conforme o Atlas do Desenvolvimento Humano no Brasil (2013), nos anos $1991 \mathrm{e}$ 2000 dos 52 municípios do estado de Rondônia, 49 apresentaram Índice de Gini maior ou igual a 0,50. Enquanto a média dos municípios brasileiros foi de 0,53.

No ano 1991, Buritis $(0,39)$, Cacaulândia $(0,44)$, Parecis $(0,44)$ São Felipe d'Oeste $(0,48)$ apresentaram índices superiores ao do Estado de Rondônia em 2011, comparativamente. No período de 2000, os municípios de Pimenteiras (0,44), Santa Luzia d'Oeste $(0,48)$ e Primavera de Rondônia $(0,49)$ apresentaram índices superiores aos do estado de Rondônia para o período de 2011, comparativamente. Para o período de 2011, todos os dez municípios melhores classificados apresentaram índices superiores ao do estado de Rondônia, comparativamente, o que denota uma melhoria considerável em termos de desigualdade (QUADRO 6). 
Quadro 6-Os dez melhores e piores municípios classificados, por Coeficiente de Gini, em

Rondônia para os anos de 1991, 2000 e 2010

\begin{tabular}{|c|c|c|c|c|}
\hline Classif. & Municipio & 1991 Municipio & 2000 Municipio & 2010 \\
\hline 10. & BURITIS & 0,39 PIMENTEIRAS DO OESTE & 0,44 TEIXEIRÓPOLIS & 0,43 \\
\hline 20. & CACAULÂNDIA & 0,44 SANTA LUZIA D'OESTE & 0,48 CANDEIAS DO JAMARI & 0,47 \\
\hline 30. & PARECIS & 0,44 PRIMAVERA DE RONDÔNIA & 0,49 CHUPINGUAIA & 0,47 \\
\hline 40. & SÃO FELIPE D'OESTE & 0,48 ALTO ALEGRE DOS PARECI & 0,51 ITAPUÃ DO OESTE & 0,47 \\
\hline 50. & CAMPO NOVO DE RONDÔI & 0,50 CANDEIAS DO JAMARI & 0,52 MONTE NEGRO & 0,47 \\
\hline 60. & TEIXEIRÓPOLIS & 0,50 SÃO MIGUEL DO GUAPORÉ & 0,53 NOVA UNIÃO & 0,47 \\
\hline 70. & VALE DO PARAÍSO & 0,50 MIRANTE DA SERRA & 0,53 SÃO FELIPE D'OESTE & 0,47 \\
\hline 80. & RIO CRESPO & 0,51 SÃO FELIPE D'OESTE & 0,53 CORUMBIARA & 0,48 \\
\hline 9o. & NOVA MAMORÉ & 0,51 SÃO FRANCISCO DO GUAPO & 0,53 OURO PRETO DO OESTE & 0,48 \\
\hline 10o. & CASTANHEIRAS & 0,51 TEIXEIRÓPOLIS & 0,53 COLORADO DO OESTE & 0,49 \\
\hline$\ldots$ & $\ldots$ & $\ldots$ & $\ldots$ & \\
\hline 42o. & ALTO ALEGRE DOS PAREC & 0,64 NOVO HORIZONTE DO OEST & 0,63 SÃO FRANCISCO DC & 0,56 \\
\hline 430. & COSTA MARQUES & 0,65 PARECIS & 0,63 CACOAL & 0,57 \\
\hline 440. & CACOAL & 0,66 ALVORADA D'OESTE & 0,65 THEOBROMA & 0,57 \\
\hline 450. & ITAPUÃ DO OESTE & 0,66 BURITIS & 0,65 VALE DO ANARI & 0,57 \\
\hline 460. & SERINGUEIRAS & 0,69 CASTANHEIRAS & 0,65 ALTA FLORESTA D'OESTE & 0,58 \\
\hline 47o. & CABIXI & 0,70 ITAPUÃ DO OESTE & 0,65 RIO CRESPO & 0,58 \\
\hline 480. & GOVERNADOR JORGE TEL & 0,70 NOVA MAMORÉ & 0,66 VALE DO PARAÍSO & 0,59 \\
\hline 49o. & CANDEIAS DO JAMARI & 0,71 MINISTRO ANDREAZZA & 0,66 MIRANTE DA SERRA & 0,60 \\
\hline $50 \mathrm{o}$. & SÃO FRANCISCO DO GUAF & 0,71 CAMPO NOVO DE RONDÔNL & 0,67 SÃO MIGUEL DO GUAPORI & 0,62 \\
\hline 510. & VALE DO ANARI & 0,75 CEREJEIRAS & 0,69 SERINGUEIRAS & 0,63 \\
\hline 520. & MINISTRO ANDREAZZA & 0,78 RIO CRESPO & 0,75 CAMPO NOVO DE RONDÔI & 0,67 \\
\hline
\end{tabular}

Fonte: Atlas do Desenvolvimento Humano no Brasil, 2013.

Sob o enfoque de clubes de convergência, identificados de forma endógena através de modelo com efeito threshold, evidências empíricas identificam a existência de um processo de convergência da desigualdade da distribuição de renda no Brasil. A renda do trabalho demonstrou-se mais significante do que a renda oriunda das transferências governamentais para a redução da desigualdade. A média de anos de estudo contribuiu de forma significativa para a redução da desigualdade nos municípios (FERREIRA E CRUZ, 2008).

A redução da desigualdade no estado de Rondônia e para os municípios pode ser atribuída à estabilidade econômica proporcionada pelo Plano Real. Ao eliminar o desenfreado aumento dos custos de produção, tornou possível o planejamento de longo prazo. Este fator foi considerado essencial para o bom desempenho agropecuário e industrial, gerando emprego e bem-estar social. (AVANÇA BRASIL, 2010). 
Figura 1- Curvas de Lorenz de Rondônia, em 1991, 2000 e 2010.

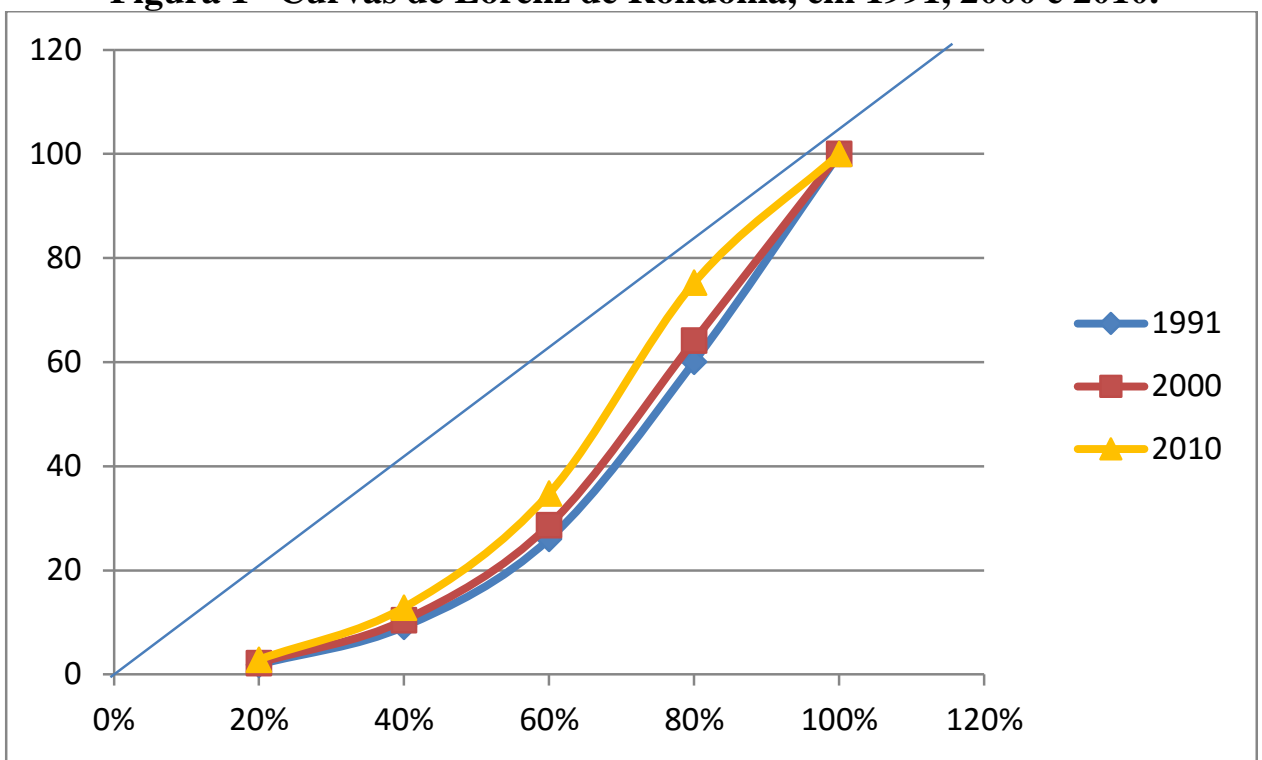

Fonte: Elaborado pelos autores (2016).

O cálculo do Índice de Gini é baseado na Curva de Lorenz. No eixo X dispõem-se os percentuais acumulados da população, sempre em ordem crescente de renda, e no eixo Y os percentuais acumulados da renda. A Curva de Lorenz de Rondônia para os períodos de 1991, 2000 e 2010 pode ser observada na Figura 1.

A área entre a reta de $45^{\circ}$ e a curva é a chamada área de concentração. Se não houvesse concentração, existiria somente a reta de perfeita igualdade. Quanto maior a concentração, maior é esta área, a curva que apresenta menor área é a curva de 2010, a que apresenta a menor área é a de 1991.

O estado de Rondônia reduziu o número de pobres em mais de $40 \%$ entre 1995 e 2008, classificando o estado como $15^{\circ}$ estado com menor número de pessoas em situação de pobreza, mas de acordo com o IPEA (2010), precisa gerar mais empregos e desenvolver melhor seus projetos sociais se quiser erradicar a pobreza até 2016. Por oportuno, segundo projeções do IPEA (2010), no ano de 2016 o estado registraria somente $4 \%$ da população vivendo em estado de pobreza, o que aproximaria a curva de Lorenz da reta de $45^{\circ}$.

\section{CONSIDERAÇÕES FINAIS}

Para atingir o objetivo de apresentar os principais indicadores sociais do estado de Rondônia, considerou-se desde os conceitos básicos de desenvolvimento humano e 
indicadores, bem como os métodos de mensuração da pobreza e desigualdade de renda, destacando as aplicabilidades destes métodos.

Baseado nos dados de 2001 a 2011, da PNAD, Censo Demográfico e Atlas do Desenvolvimento Humano no Brasil, observou-se melhora no acesso a renda e redução na proporção de pobres no estado de Rondônia. Contudo ainda existem pessoas abaixo da linha da pobreza neste estado e muitas em situação de pobreza extrema.

No Brasil, a utilização de indicadores sociais, configura-se em discussão recente, porém, tornando-se cada vez mais evidente a sua importância para o processo de gestão, isso porque possibilitam informações importantes, permitindo avaliar o passado, o presente e de que forma seguir, em relação aos valores e alcance dos objetivos previamente identificados.

Os dados estatísticos e informações de renda e crescimento econômico são úteis para a construção dos indicadores, na verdade, são matérias-primas para estruturar indicadores. Contudo basear as decisões de gestão em estatísticas públicas, que são simples junções de dados, sem devida contextualização e interpretação dos conceitos operacionalizados, pode-se incorrer no velho erro de desconsiderar a qualidade de vida e o desenvolvimento humano como elemento importante em uma gestão.

\section{REFERENCIAS}

ALMEIDA, M. A. B. Qualidade de vida: definição, conceitos e interfaces com outras áreas, de pesquisa. São Paulo: Escola de Artes, Ciências e Humanidades - EACH/USP, 2012.

ATLAS do Desenvolvimento Humano e Condições de vida: Indicadores Brasileiros. PNUD/IPEA/FJP, 2013.

AVANÇA BRASIL. Ações Federais de 1995 a 2002: Rondônia. Disponível em < http://www.abrasil.gov.br/estados/index.htm>, acesso em 18 set. 2013.

BARBOSA, S. R. C. S. Qualidade de Vida e ambiente: uma temática em construção. In: BARBOSA, Sônia Regina da Cal Seixas (org.). A temática ambiental e a pluralidade do Ciclo de Seminários do NEPAM. Campinas: UNICAMP, NEPAM, p. 401-423, 1998.

BORBA, Ana Emília de Oliveira. Proposta de indicadores de sustentabilidade para o setor da construção civil. 2009. Dissertação Mestrado. 134 f. Universidade de Pernambuco, Escola Politécnica, Recife. 2009.

BVS - Biblioteca Virtual em Saúde. Características dos indicadores Socioeconômicos. 2011. Disponível em:

< http://www.ripsa.org.br/fichasIDB/record.php?node=B.5\&lang=pt>, acesso em 11 set. 2013

COLE, R. Sustainable Building: Indicators of progress. Sustainable Building, n. 4, p. 17, 2002. 
COSTA, R. D. Principais Índices de Pobreza Brasileira. Revista Eletrônica. Faculdade de Educação, Administração e Tecnologia de IBAITI. Volume 8, 2010. . Disponível em:

$<$ http://www.feati.edu.br/revistaeletronica/downloads/numero1/principaisIndicesPobrezaBrasileira.pdf $>$, acesso em 11 set. 2013

FERREIRA, R. T.; CRUZ, M. S.. Clubes de Convergência na Desigualdade de Renda nos Municípios Brasileiros. XXXVI Encontro nacional de economia. Associação Nacional dos Centros de Pós-Graduação em Economia (ANPEC). Salvador, Bahia, 2008. Disponível em:

<http://www.anpec.org.br/encontro2008/artigos/200807171159380-.pdf>, acesso em 11 set. 2013

HARDI, P; BARG, S. Measuring sustainable development: review of current practice. Winnipeg: IISD, 1997

HOEHN, John R.; BERGER, Mark C.; BLOMQUIST, Glenn C.. A Hedonic Model of Interregional Wage, Rents, and Amenity Values. Journal of Regional Science 29(4): 605620. 1987.

IPEA-Instituto de Pesquisa Econômica Aplicada. Dimensão, evolução e projeção da pobreza por região e por estado no Brasil. Comunicados do Ipea $\mathrm{N}^{\circ}$ 58. 2010

IPECE- Instituto de Pesquisa e Estratégia Econômica do Ceará. Entendendo o Índice de GINI. Governo do Estado do Ceará, 2010. Disponível em:

< http://www.ipece.ce.gov.br/publicacoes/Entendendo_Indice_GINI.pdf>, acesso em 11 set. 2013

JANNUZZI, Paulo de Martino. Indicadores sociais no Brasil. Campinas, SP ; Editora Alinea, 2001.

KAHN, M. A revealed preference approach for ranking city quality life. Journal of Urban Economics, v. 38, p. 221-235, 1995.

KNAPP, T. A.; GRAVES, P. E.. "On the Role of Amenities in Models of Migration and Regional Development.” Journal of Regional Science 29(1): 71-87. 1989.

KLIKSBERG, Bernardo. Falácias e mitos do desenvolvimento social. Cortez Editora. São Paulo-SP, 2001.

KIM, Kwang-Koo; MARCOUILLER, David W.; DELLE, Steven C. Natural Amenities and Rural Development: Understanding Spatial and Distributional Attributes. Growth and change: A journal of urban and regional policy. - Lexington, Ky.: University of Kentucky, ISSN 0017-4815, ZDB-ID 2827645. - Vol. 36. 2005, 2, p. 273-297. 2005

MARANS, R.W.; STIMSON, Robert J. Investigating Quality of Urban Life Theory, Methods, and Empirical Research. Social Indicators Research Series, Vol 45. London New York: Springer, 2011.

MARQUES, J. F.; COMUNE, A. Quanto vale o ambiente: interpretações sobre o valor econômico ambiental In: ENCONTRO NACIONAL DE ECONOMIA, 23., Salvador, BA, 12 a 15 de dezembro de 1995, Anais p.633-651.

MARZALL, Kátia. Indicadores de sustentabilidade para agroecossistemas. UFRGS, Porto Alegre/RS, Tese

MARZALL, Kátia e ALMEIDA, Jalcione. INDICADORES DE SUSTENTABILIDADE PARA AGROECOSSISTEMAS Estado da arte, limites e potencialidades de uma nova 
ferramenta para avaliar o desenvolvimento sustentável. Cadernos de Ciência \& Tecnologia, Brasília, v.17, n.1, p.41-59, jan./abr. 2000.

McGRANAHAN, David A., Natural Amenities Drive Rural Population Change. Food and Rural Economics Division, Economic Research Service, U.S. Department of Agriculture. Agricultural Economic Report. Nº. 781. Washington, 1999.

MELO, L. F. Metrópole, Tecnologia, Amenidades e Riscos. Núcleo de Estudos de População - NEPO/Unicamp. III Encontro da ANPPAS, Brasília - DF, 2006.

MOTTA, R. S.. Manual de valoração econômica de recursos ambientais. Brasília: MMA, 1998.

MINAYO, M. C. S.; HARTZ, Z. M. A.; BUSS, P. M. Qualidade de Vida e saúde: um debate necessário. Ciência \& Saúde Coletiva. Rio de Janeiro, v. 5, n.1, 2000, p. 7-18.

NAHAS, M. I. P. PEREIRA, M. A. M.; ESTEVES, O. A.; GONÇALVES, E. Metodologia de construção do Índice de Qualidade de Vida Urbana dos municípios brasileiros (IQVU-BR). In: XV Encontro Nacional de Estudos Populacionais da Associação Brasileira de Estudos Populacionais., 2006, Caxambu, MG. XV Encontro Nacional de Estudos Populacionais da Associação Brasileira de Estudos Populacionais. 2006.

NEDER, Henrique Dantas. Analise de Indicadores Sociais Utilizando o Stata. Instituto de Economia - Universidade Federal de Uberlandia. 2013. Disponível em < http://www.ecn26.ie.ufu.br/TEXTOS_ESTATISTICA/ANALISE_POLITICAS_SOCIAIS.pdf >, acesso em 11 set. 2013.

PEREIRA, Érico Felden; TEIXEIRA, Clarissa Stefani and SANTOS, Anderlei dos. Qualidade de vida: abordagens, conceitos e avaliação. Rev. bras. educ. fís. esporte [online]. 2012, vol.26, n.2, pp. 241-250. ISSN 1807-5509.

PNUD- Programa das Nações Unidas para o Desenvolvimento. Informe sobre Desarrollo Humano 1996. Ediciones Mundi-Prensa, 1996.

Relatório do Desenvolvimento Humano 2011 Sustentabilidade e Equidade: Um Futuro Melhor para Todos. Plaza, New York, 2011.

Relatório do Desenvolvimento Humano 2013. A Ascensão do Sul: Progresso Humano num Mundo Diversificado. Plaza, New York, 2013.

REIS, E. J.; MOTTA, R. S. The aplication of economic instruments in environmental policy: the Brazilian Case. Revista Brasileira de Economia, v.. 48, n 4, out./dez. 1994.

ROBACK, J. The value of local urban amenities: theory and measurement. Ph.D. Dissertation, University of Rochester, 1980.

ROCHA, R. M.; MAGALHÃES, A. M. As amenidades naturais influenciam a escolha locacional dos trabalhadores? Evidências para as regiões metropolitanas brasileiras. R. bras. Est. Pop., Rio de Janeiro, v. 28, n. 2, p. 369-387, jul./dez. 2011.

ROCHA, R. M.; Amenidades locais versus oportunidades econômicas: um ranking da qualidade das amenidades para as regiões metropolitanas do Brasil. Associação Nacional dos Centros de Pósgraduação em Economia [ANPEC]. 2007. Disponível em < http://www.anpec.org.br/encontro2007/artigos/A07A118.pdf >, acesso em 18 jun. 2013.

ROCHA, R. M.; MAGALHÃES, A.M. Qualidade das amenidades urbana e a heterogeneidade das preferências: um estudo para as regiões metropolitanas brasileiras. 
2010. Disponível em < http://aplicativos.fipe.org.br/enaber/pdf/70.pdf >, acesso em 28 jun 2013.

As amenidades naturais influenciam a escolha locacional dos trabalhadores? Evidências para as regiões metropolitanas brasileiras. Revista brasileira de Estudos de População, Rio de Janeiro, v. 28, n. 2, p. 369-387, jul./dez. 2011.

Valoração das amenidades urbanas: uma estimação a partir dos diferenciais salariais e do custo de habitação para as regiões metropolitanas brasileiras. Rev. econ. contemp. [online]. 2013, vol.17, n.1, pp. 69-98. ISSN 1415-9848. 2013.

ROCHA, Sonia. Pobreza no Brasil. Afinal, do que se trata? 3. ed. Rio de Janeiro: Editora FGV, 2008.

Sonia. Pobreza no Brasil: A Evolução de Longo Prazo (1970-2011). XXV Fórum Nacional: O Brasil de Amanhã. Transformar Crise em Oportunidade. Rio de Janeiro, 2013.

SANTOS, L. D.;MARTINS I. A qualidade de vida urbana o caso da Cidade do Porto. Working papers da FEP. Porto: Universidade Federal do Porto. Investigação - Trabalhos em curso, $\mathrm{n}^{\mathrm{o}}$ 116, 2002.

SILVEIRA NETO, R. M.; MENEZES, T. A.. Preferência revelada e arbitragem espacial: determinando um ranking de qualidade de vida para as regiões metropolitanas do Brasil. Rev. Bras. Econ. [online]. 2008, vol.62, n.4, pp. 361-380. ISSN 0034-7140.

STEINBERG, M. W. 2000. Making sense of environmental justice. Forum for Applied Research and Public Policy Fall:82.89. 2000.

WEINBERG, A. S. 1998. The environmental justice debate: new agendas for a third generation of research. Society and Natural Resources 11:605. 1998.

WONG, C. Developing indicators to inform local economic development. Urban Studies, 39, 1833-1863. 2002.

VELEVA, V.; ELLENBECKER, M. A proposal for measuring business sustainability: addressing shortcomings in existing frameworks. University of Massachusetts Lowell, USA. 2000.

YWATA, S. Y., GIROTO, A. P. S., Os Indicadores Sociais como Instrumento de Gestão Social. Serviço Social em Revista. Volume 10 - Número 2. Jan/Jul 2008. Disponível em <http://www.uel.br/revistas/ssrevista/c-v10n2_valderes.htm>, acesso em 18 set. 2013. 